\title{
O USO DE NANOTECNOLOGIAS NA PRODUÇÃO DE MEDICAMENTOS: O ESTADO DA ARTE DA REGULAÇÃO NO BRASIL
}

\author{
THE USE OF NANOTECHNOLOGY IN THE PRODUCTION OF MEDICINES: THE \\ STATE OF THE ART REGULATION IN BRAZIL
}

\begin{abstract}
GABRIELLE JACOBI KöLLING
Bacharel em Direito (Unisinos). Mestra em Direito Público (Unisinos); especialista em Direito Sanitário (ESPRS e Universidade de Roma Tre). Doutoranda em Direito Público (Unisinos, bolsista CAPES). Pesquisadora do Direito nas áreas: Sociologia Jurídica - Teoria dos Sistemas Sociais; Teoria Geral do Direito; Direito Constitucional e Direito Sanitário. Trabalha especificamente com Democracia, Saúde Pública e Políticas Públicas de Saúde. Membro da Red Iberoamericana de Derecho Sanitario (Fiocruz/ Brasília). Professora Universitária. Foi pesquisadora colaboradora do Programa de Direito Sanitário da Fundação Oswaldo Cruz (Fiocruz - Brasília/DF). Professora Universitária e Coordenadora Adjunta do Curso de Direito da Universidade Luterana do Brasil koll.gabrielle@gmail.com
\end{abstract}

SANDRA REgINA MARTINI

Possui graduação em Ciências Sociais pela Universidade do Vale do Rio dos Sinos (1983), mestrado em Educação pela Pontifícia Universidade Católica do Rio Grande do Sul (1997), doutorado em Evoluzione dei Sistemi Giuridici e Nuovi Diritti pela Università Degli Studi di Lecce (2001), Pós-doutorado em Direito (Roma Tre, 2006) e Pós-doutorado em Políticas Públicas (Universidade de Salerno, 2010). Atualmente é professora titular da Universidade do Vale do Rio dos Sinos, da Scuola Dottorale Internazionale Tullio Ascarelli e professora visitante da Universita Degli Studi Di Salerno. Foi diretora da Escola de Saúde Pública do Rio Grande do Sul (janeiro de 2007 a fevereiro de 2011), foi membro( de janeiro de 2008 a dezembro de 2013) do Conselho Superior da Fundação de Amparo à Pesquisa do Estado do Rio Grande do Sul (FAPERGS). Atualmente é pesquisadora gaúcha FAPERGS, e pesquisadora produtividade CNPq. É avaliadora do Basis do Ministério da Educação e Cultura e do Basis do Instituto Nacional de Estudos e Pesquisas Educacionais Anísio Teixeira. Tem experiência na área de Direito, com ênfase em sociologia jurídica, atuando principalmente nos seguintes temas: saúde pública, políticas públicas, sociologia jurídica e sociedade srmvial@terra.com

\section{RESUMO}

A utilização de nanotecnologias na sociedade atual é uma realidade bastante invisível que a ciência, o direito, a política e a saúde ainda não “dominam”. No entanto, o direito ainda não apresenta soluções do tipo jurídicas para regular essa tecnologia e os riscos oriundos do seu uso ainda são desconhecidos. 0 sistema da política ainda não tomou decisões coletivamente vinculantes sobre a regulação do uso de tecnologia nano na produção de medicamentos, no entanto, já existem medicamentos sendo produzidos e comercializados com partícula nano e os seus riscos são desconhecidos e não monitorados. Assim, a pretensão do artigo é analisar o contexto da regulação do uso de tecnologia nano na produção de medicamentos no Brasil, no cenário de regulação, estabelecendo uma discussão a partir da pluralidade de fontes do direito como possibilidade para a regulação.

Palavras-chave: saúde; regulação; nanotecnologia; risco.

\begin{abstract}
The use of nanotechnology in society today is a very invisible reality that science, law, politics and health did not "dominate". However, the law still does not have the legal type solutions to regulate this technology and the risks arising from its use are still unknown. The political system has not yet taken collectively binding decisions on the regulation of the use of nanotechnology in the production of medicines, however, there are already drugs being produced and marketed with nano particle and its risks are unknown and unmonitored. Thus, the claim of the paper is to analyze the context of the regulation of the use of nanotechnology in the production of medicines in Brazil, regulatory scenario, establishing a discussion from the plurality of sources of law as a possibility for regulation.
\end{abstract}

Keywords: health; regulation; nanotechnology; risk. 

MEDICAMENTOS: O ESTADO DA ARTE DA REGULAÇÃO NO BRASIL

\section{SUMÁRIO}

INTRODUÇAO; 1 ASPECTOS INTRODUTÓRIOS ACERCA DAS NANOTECNOLOGIAS; 1.1 Contextualizando a tecnologia nano; 1.20 uso da nanotecnologia e o risco sanitário; 2 EMERGÊNCIA DA PLURALIDADE DE FONTES DO DIREITO E DE ATORES NÃO LEGISLATIVOS NA REGULAÇÃO; 3 A PRODUÇÃO DE MEDICAMENTO COM PARTÍCULAS NANO: O ESTADO DA ARTE DA (DES)REGULAÇÃO DA NANOTECNOLOGIA NA PRODUÇÃO DE MEDICAMENTOS NO BRASIL; CONCLUSÃO; REFERÊNCIAS.

\section{INTRODUÇÃO}

Esse problema tem um componente cultural - a atitude dos homens politicos e cidadãos vis-à-vis ao risco e à regulamentação'.

O documento "Éthique et Politique des Nanotechnologies" chama a atenção para o fato de que o problema da regulamentação jurídica das nanotecnologias tem um viés cultural. Esse é um problema de saúde pública e de direito que ainda não é enfrentado de modo satisfatório, tendo em vista que ainda não temos no Brasil a cultura de discutir a regulação das nanotecnologias a partir de um olhar inter ou transdisciplinar, que possibilite a interação de saber e o "manuseio" do fenômeno do risco. Assim, é essencial que haja convivência entre as áreas duras e brandas (física, química, tecnologia etc. e direito, respectivamente), conforme Engelmann ${ }^{2}$ nos adverte, pois assim será possível a transdisciplinaridade, por meio da linguagem, o que representará uma abertura epistemológica (necessária).

A (r)evolução tecnológica oferta à sociedade a possibilidade de inovação, e isso não é diferente na produção de medicamentos. Já existem medicamentos produzidos com tecnologia nano. O uso dessa tecnologia está vinculado ao futuro incerto. "Tudo é incerto e derradeiro. Tudo é disperso, nada é inteiro" ${ }^{3}$, o conhecimento acerca das nanotecnologias e dos seus efeitos não é inteiro, é incerto e inseguro, estamos na era do risco, da incerteza. 0 estudo das nanotecnologias é igualmente incerto e não pode ser realizado a partir de uma totalidade, mas

\footnotetext{
${ }^{1}$ UNESCO. Organisation des Nations Unies pour l'éducation, la science et la culture. Éthique et Politique des Nanotechnologies. Paris, $2007 . \quad$ Disponível http: / / unesdoc.unesco.org/images/0014/001459/145951f.pdf . Acesso em 23/08/2015.

2 VON HOHENDORFF, Raquel; ENGELMANN, Wilson. Nanotecnologias aplicadas aos agroquímicos no Brasil. A gestão dos riscos a partir do diálogo entre as fontes do direito. Curitiba: Juruá Editora, 2014.

3 PESSOA, Fernando. Mensagem. Disponível em: http://www.cfh.ufsc.br/ magno/mensagem.htm . Acesso em 28 ago. 2015.
} 
sim de modo fragmentado, dada a complexidade das nanopartículas. 0 direito regulatório ainda não consegue dar respostas do tipo jurídicas, na sua atual estrutura.

Assim, com esse artigo pretende-se analisar o estado da arte da regulação do uso de tecnologia nano na produção de medicamentos no Brasil a partir de algumas categorias sistêmicas, bem como de contribuições oriundas de outras teorias que serão oportunas para discussões específicas acerca das fontes do direito.

No que concerne à metodologia é oportuno destacar que se utilizará o método de abordagem descritivo-analítico e a técnica de pesquisa será a bibliográfica.

0 artigo está dividido em três partes, inicialmente contextualizaremos as nanotecnologias no cenário brasileiro e a sua relação com o risco sanitário. Posteriormente, a necessidade ou emergência da pluralidade de fontes no direito e de atores não legislativos na regulação e, por fim, o estado da arte da (des)regulação no país.

\section{APORTES INTRODUTÓRIOS ACERCA DAS NANOTECNOLOGIAS NO CENÁRIO ATUAL}

Nesse item apresentaremos um breve panorama no que tange às nanotecnologias no contexto brasileiro. Primeiramente, abordaremos a tecnologia nano numa perspectiva mais conceitual, posteriormente, a relação do uso desse tipo de tecnologia na produção de medicamentos com o risco sanitário.

\subsection{Contextualizando a Tecnologia nano}

O termo nanotecnologia está vinculado à manipulação de matéria em escala molecular. Pode-se dizer que as nanotecnologias estão calcadas no desenvolvimento de procedimentos que fazem uso de nanomateriais. Esses materiais possuem uma dimensão inferior a 100 nanômetros $(10 \mathrm{mn})^{12^{4}}$.

As nanotecnologias são marcadas, dentre outras características, pela multidisciplinaridade, no que diz respeito as suas técnicas. Essa "qualidade" é o que possibilita

${ }^{4}$ VON HOHENDORFF, Raquel; ENGELMANN, Wilson. Op Cit., 2014. 
dominar partículas com dimensões muito pequenas que apresentam propriedades mecânicas, óticas, magnéticas e químicas, constantemente inovadoras ${ }^{5}$.

Nota-se, pois, que quando o assunto é nanotecnologia as possibilidades conceituais são abrangentes. Pode-se compreender a tecnologia nano como controle da matéria e os processos em nanoescala, tipicamente, mas não exclusivamente, inferiores a 100 nanômetros em uma ou mais dimensões, em que o aparecimento de fenômenos dependentes do tamanho, geralmente, permite novas aplicações, ou ainda; a utilização das propriedades dos materiais em nanoescala, que são diferentes das propriedades dos átomos e moléculas individuais e matérias a granel, para criar materiais, dispositivos e sistemas melhores que exploram essas novas propriedades ${ }^{6}$.

A nanotecnologia pode ser aplicada em praticamente todas as áreas da sociedade, o que demanda interdisciplinaridade para sua elaboração e integração de diversas disciplinas; é uma tecnologia que demanda epistemologia transdisciplinar e multidisciplinar para sua compreensão, pois tem diferentes segmentos de aplicação na sociedade, há uma junção de saberes para analisar o uso da tecnologia nano, bem como os seus riscos em áreas distintas. Considerando tais características, a nanotecnologia é considerada a via para a resolução de muitos dos problemas da atualidade. ${ }^{7}$

A nanociência e a nanotecnologia ( $N \& N$ ), o estudo e a aplicação dessa tecnologia, respectivamente, ganham espaço no cenário brasileiro a partir dos anos 2000, quando ocorreram as primeiras iniciativas de reunir pesquisadores que já atuavam ou que tinham interesse na área. ${ }^{8}$ No entanto, a discussão da regulação, na ótica jurídica, ainda é recente, incipiente e disciplinar.

Observa-se que, no Brasil, há prioridade em relação ao desenvolvimento da nanotecnologia em mercados específicos, são três: produtos farmacêuticos, químicos e cosméticos. Esses mercados são influenciados pelo consumo do mercado farmacêutico, pelo desenvolvimento interno do setor químico e farmacêutico, bem como pela biodiversidade. ${ }^{9}$

\footnotetext{
${ }^{5}$ VON HOHENDORFF, Raquel; ENGELMANN, Wilson. Op Cit., 2014.

${ }^{6}$ FRONZA, T.; GUTERRES, S. S.; POHLMANN, A. R.; TEIXEIRA, H.F. Nanocosméticos: em direção ao estabelecimento de marcos regulatórios. Porto Alegre: Gráfica da UFRGS, 2007.

${ }^{7}$ MEDEIROS, E.S.; PATERNO L.G.; MATTOSO L.H.C. Nanotecnologia. In: Durán N, Mattoso LHC, Morais PC, organizadores. Nanotecnologia Introdução, preparação e caracterização de nanomateriais e exemplos de aplicação. São Paulo: Artliber, 2006.

${ }^{8}$ FERNANDES, M.F.M., FILGUEIRAS CAL. Um panorama da nanotecnologia no Brasil (e seus macro-desafios). Quim. Nova, v. 8, n. 31, 2008, p.2205-2213.

9 BRASIL, ANVISA. Agência Nacional de Vigilância Sanitária. Gestão 2005-2010: principais realizações / Agência Nacional de Vigilância Sanitária. Brasília: ANVISA, 2010.
} 
O setor da indústria farmacêutica tem investido na pesquisa e no desenvolvimento de novos medicamentos nanotecnológicos, com sistemas nanoestruturados de liberação de fármacos. Um dos resultados dessas novas possibilidades de liberação dos fármacos são as vantagens da redução dos efeitos adversos e da toxicidade do fármaco, proporcionando, assim, maior conforto ao paciente e maior adesão ao tratamento. Todavia, esses sistemas também podem apresentar algumas desvantagens, tais como: 1) toxicidade dos produtos ou dos metabólitos oriundos da sua biodegradação; 2) preço mais elevado, devido ao custo do processo tecnológico, que pode ser compensado pela redução das doses; e, 3) possibilidade de permear barreiras biológicas e atingir órgãos nobres como o cérebro. ${ }^{10}$

É oportuno destacar que o Brasil, desde o ano de 2010, aparece em $13^{\circ}$ lugar no ranking dos países que têm prioridade para depósito de patentes relacionadas à nanotecnologia. Desde o ano de 2008, ocupa essa mesma posição entre aqueles com preferência para depósito de patentes relacionadas à nanobiotecnologia ${ }^{11}$. Esse contexto revela o forte papel da sociedade brasileira, no desenvolvimento de produtos ou processos relacionados à nanotecnologia e à nanobiotecnologia, ou ainda, o interesse das empresas estrangeiras no mercado nacional ${ }^{12}$. Isso reforça ainda mais a necessidade urgente de regulação.

É relevante observarmos os resultados da análise documental realizada pela Vigilância Sanitária Brasileira sobre medicamentos que utilizaram nanotecnologia. Uma das conclusões do estudo, publicado na forma de artigo, foi a de que, apesar da incipiente regulação desses medicamentos, foram encontrados 07 registrados no Brasil e sem identificação clara e objetiva na bula e na embalagem quanto as suas constituições nanotecnológicas, e 04 como medicamentos similares, até $2014^{13}$.

\footnotetext{
${ }^{10}$ BATISTA, Ariane de Jesus Sousa. PEPE, Vera Lúcia Edais. Os desafios da nanotecnologia para a vigilância sanitária de medicamentos. Ciênc. saúde coletiva, v.19, n.7, Rio de Janeiro, Julho de 2014. Disponível em: http://dx.doi.org/10.1590/1413-81232014197.02462013. Acesso em 13 jun. 2015.

11 NUNES, João Pimentel Rohem-Santos P. Pedidos de Patente sobre Nanotecnologia publicados no $2^{\circ}$ semestre de 2009. INPI/DART/CEDIN/DIESPRO. Alerta Tecnológico n²0; 2010a. Disponível em: http://www.inpi.gov.br/menuesquerdo/informacao/alerta_nano_20.pdf.

Acesso em: 10/05/2015.

${ }_{12}$ ROSSI-BERGMAN, B. A nanotecnologia: da saúde para além do determinismo tecnológico. Cienc Cult, v. 2, n. 60, 2008, p.54-57.

${ }^{13}$ BATISTA; PEPE, Op Cit., 2014.
} 
No entanto, a gerência da ANVISA divulgou que foram encontrados 10 medicamentos que fazem referência à nanotecnologia registrados na Agência entre os produtos. (BRASIL, 2014.) Observa-se que não há sequer coerência entre as informações da VISA ${ }^{14}$.

\subsection{0 uso de nanotecnologia e o risco sanitário}

A (r)evolução tecnológica, em especial aquela que concerne às nanotecnologias, desencadeia consequências éticas, jurídicas e sociais na sociedade contemporânea. Barreto ${ }^{15}$ nos adverte que o uso da capacidade humana de criar e produzir em grande escala faz com que as ações da sociedade tecnocientífica provoquem, crescentemente, efeitos nocivos que são inseparáveis dos efeitos bons.

Uma das características das nanotecnologias é a incerteza em relação aos seus efeitos ( bons e maus $\left.^{16}\right)$. Essa incerteza afeta também o direito, e esse, por sua vez, coloca em discussão os seus pilares da previsibilidade e certeza. Os estudos existentes na atualidade ainda não permitem ou possibilitam sabermos quais são os reais efeitos dos nanocompostos em relação à saúde dos seres humanos ${ }^{17}$. Assim, cabe um olhar atento do direito no que se refere ao risco contido nessa relação do uso das nanotecnologias, na produção de medicamentos, com a regulação jurídica. Como regular ou gerenciar o risco?

São oportunas as considerações de $\operatorname{Aith}^{18}$ no que tange ao objetivo do direito sanitário: reduzir os riscos de doenças e de outros agravos à saúde da população. 0 autor vai além: é, também, tarefa do direito sanitário orientar os poderes públicos a adotarem medidas concretas que possibilitem visualizar os possíveis riscos à saúde e as consequentes medidas cabíveis. Notase, pois, que é tarefa do direito sanitário gerenciar o risco oriundo do uso de tecnologia nano.

\footnotetext{
${ }^{14}$ É oportuno destacar que o termo VISA (Vigilância Sanitária) é atividade da ANVISA, logo, é comum na literatura sanitária utilizar ambas as expressões para fazer referência à atividade de vigilância sanitária.

${ }^{15}$ BARRETO, Vicente de Paulo. O Fetiche dos Direitos Humanos e outros temas. 2 ed. Porto Alegre: Livraria do Advogado, 2013.

${ }^{16}$ Grifo da autora.

${ }^{17}$ VON HOHENDORFF; ENGELMANN, Op Cit., 2014.

${ }_{18} \mathrm{AITH}$, Fernando. Curso de Direito Sanitário - a proteção do direito à saúde no Brasil. São Paulo: Quartier Latin, 2007.
} 
Quando o assunto é nanotecnologia ainda estamos num terreno fértil e bastante desconhecido, especialmente no que concerne aos riscos oriundos do uso dessa tecnologia. Não sabemos quais são os riscos e tampouco temos um acompanhamento contínuo para avaliação dos riscos (tarefa que é da Vigilância Sanitária - monitoramento dos riscos).

A presença do risco coloca em cheque as certezas, insere-nos num universo de incertezas. Vejamos as contribuições de Maturana e Varela ${ }^{19}$ a esse respeito:

[...] tendemos a viver num mundo de certezas, de uma perspectividade sólida e inquestionável, em que nossas convicções nos dizem que as coisas são da maneira como as vemos e que não pode haver alternativa ao que parece certo. Tal é nossa situação cotidiana, nossa condição cultural, nosso modo corrente de sermos humanos.

O paradigma da certeza já se tornou uma ficção, o que nos resta é aceitar e trabalhar com as probabilidades diante do risco. A nossa tendência é a busca de uma certeza. Entretanto, o risco balança as estruturas dessa sólida e inquestionável certeza. O direito tem como uma de suas tarefas, gerenciar o risco. Essa função de "gerenciador de riscos" é notória no campo do uso de tecnologia nano na produção de medicamentos, já que o direito sanitário tem a pretensão de gerir os riscos dentro da saúde.

Conforme De Giorgi ${ }^{20}$, o controle das indeterminações sempre se mostrou como problema para a sociedade. As estruturas da sociedade moderna são paradoxais. Na contemporaneidade reforçam-se, de modo simultâneo, a segurança e a insegurança, a determinação e a indeterminação, dentre outros paradoxos. Nesse contexto, o risco é uma condição estrutural para os sistemas, notadamente em relação ao direito, tendo em vista que o risco sobrecarrega o direito que fixará pressupostos jurídicos que permitam a orientação das expectativas no futuro (incerto, impreciso). E é justamente essa orientação em relação às expectativas no futuro, que falta ao direito, quando o assunto é o uso de nanotecnologia na produção de medicamentos.

Assim, observa-se que na sociedade atual, o sistema do direito tem que dar respostas que ultrapassem as tradicionais. Logo, percebe-se que novas demandas (tais como a regulação do uso de tecnologia nano na produção de medicamentos) vão surgindo e o direito fundamentado na perspectiva dogmática não responde às demandas da sociedade. Esses problemas novos só podem ser afrontados por meio de uma análise da complexidade típica da sociedade moderna, a

\footnotetext{
${ }^{19}$ MATURANA, H. R.; VARELA, F. J. A árvore do conhecimento: as bases biológicas do comportamento humano. Tradução de Humberto Mariotti. São Paulo: Athenas, 2011. p. 11.

${ }^{20}$ DE GIORGI, Raffaele. 0 risco na sociedade contemporânea. Tradução de Cristiano Paixão. Revista CCJ UFSC, n. 28, ano 15, junho de 1994, p. 45-54.
} 
qual permite observar e desvelar os seus paradoxos. As contingências da modernidade tornam-na cada vez mais complexa e, ilimitadamente, mutável, o que não é diferente no direito sanitário e nas nanotecnologias, pois, afinal, a aplicação de nanotecnologias na produção de medicamentos gera novos medicamentos. Tem-se, ainda, a possibilidade de medicamentos drug delivery ${ }^{21}$, terapias medicinais, dentre outras possibilidades, mas todas com grande complexidade, risco e mutabilidade.

“Conhecer e pensar não é chegar a uma verdade absolutamente certa, mas dialogar com a incerteza" 22 . Dialogar com a incerteza, no dizer de Morin, remete-nos à sociedade cuja característica (uma delas) é o risco, na qual já não há mais espaço para as certezas. E é nesse contexto da sociedade moderna que estão o direito sanitário e as nanotecnologias. 0 direito precisa dar respostas jurídicas aos riscos, e essas surgem a partir da irritação que o sistema jurídico sofre.

Atualmente, quando o assunto é ciência, as dúvidas colocam-se à frente das certezas. A inovação da nanotecnologia faz emergir diferentes questões relacionadas a distintas áreas do conhecimento, especialmente à área jurídica, em função dos riscos oriundos do seu uso. ${ }^{23}$

A ideia de risco fragiliza, para não dizer elide, um dos alicerces do direito: a segurança jurídica. A partir do momento em que admitimos o risco não é mais possível conceber a existência da certeza ou segurança jurídica. A regulação do uso de tecnologia nano precisa considerar a incerteza oriunda do risco.

Considerando que a regulação de uma nova tecnologia é sempre um risco, especialmente no caso das nanos, pode-se cogitar uma regulação baseada em evidência científica, interativa, colaborativa e flexível, esse é um modo de enfrentar o risco. ${ }^{24}$ Explicando: não se quer dizer, com isso, que o direito não decidirá com o seu código binário (direito/não direito), mas sim que o que pode irritar o sistema são essas informações oriundas das evidências científicas.

No contexto do risco do uso de tecnologia nano para a produção de medicamentos, estima-se que, conforme os sistemas de liberação de fármacos nanoestruturados, $40 \%$ dos novos fármacos apresentam resultados biofarmacêuticas indesejáveis, ou seja, baixa solubilidade,

\footnotetext{
${ }^{21}$ No que tange ao Drug Delivery System (DDS), pode-se compreendê-lo como um sistema de administração desenvolvido para prolongar o tempo de liberação do fármaco no organismo, sustentar sua concentração plasmática e controlar a localização temporal e espacial das moléculas in vivo, por meio da aplicação de princípios biológicos e químicos (SWARBRICK, 2007).

${ }_{22}$ MORIN, Edgar. Cabeça bem-feita. Tradução Eloá Jacobina. Rio de Janeiro: Bertrand Brasil, 2008. P. 59.

${ }^{23}$ VON HOHENDORFF; ENGELMANN, Op Cit., 2014.

24 DEVALAPALLY, $\mathrm{H}$ et al. Role of nanotechnology in pharmaceutical product development. Journal of Pharmaceutical Sciences, n. 10, v. 96, n. 10, p. 2.547-2.565, 2007.
} 
baixa permeabilidade, degradação/metabolismo, complexação, influxo intestinal e transporte inadequado. ${ }^{25}$ Esse é um risco que precisa ser monitorado e considerado na regulação, inclusive como evidência.

Deve-se observar que, na questão dos nanomateriais, não temos dados sobre os seus impactos na saúde. Desconhecemos a toxicidade dos materiais nanotecnológicos, bem como os métodos para realizar os testes de toxicidade e os impactos para a saúde, oriundos da manipulação de nanopartículas. ${ }^{26}$ Essa é uma realidade que o sistema do direito precisa considerar para regular o uso da tecnologia nano na produção de medicamentos.

O elixir da morte (DDT - Dicloro-difenil-tricloretano), do livro “A primavera silenciosa”, de Rachel Carson, em 1962, que foi um dos primeiros escritos a chamar a atenção do mundo para os riscos da ciência e da tecnologia, ou seja, a relação ciência, inovação e risco não é invenção da modernidade. A presença do risco já é uma constante social há décadas, com a qual, o direito, a saúde e a política ainda não sabem lidar.

Para o enfrentamento do risco tem-se o princípio da precaução. Esse princípio apresentase como "uma concepção relativamente nova e norteadora da ação" diante de "riscos de danos graves e irreversíveis". No contexto desse princípio, não devemos "renunciar a agir" sob o "pretexto da incerteza científica" e tampouco taxá-lo de "princípio antiprogresso"27.

No cenário das nanotecnologias, é condição de possibilidade a atribuição de probabilidades às incertezas para a tomada de decisão na regulação do uso de tecnologia nano na produção de medicamentos. A probabilidade é uma medida preventiva que apresenta alguma informação e conhecimento prévios dos eventos, calculada e inserida pelo observador externo ${ }^{28}$.

Temos uma “incerteza epistêmica” em relação aos resultados oriundos do uso da tecnologia nano na produção de medicamentos, é isso é reflexo da falta de conhecimento acerca das probabilidades do evento ${ }^{29}$. É preciso que haja estudo de probabilidade dos riscos, as conclusões desse estudo serão basilares para a aplicação do princípio da precaução no sentido de diminuir ou minimizar o risco.

\footnotetext{
${ }^{25}$ DEVALAPALLY, Op Cit., 2007.

${ }^{26}$ VON HOHENDORFF; ENGELMANN, Op Cit. , 2014.

27 BOURG, D. Principe de précaution. In: Mesure S, Savidan P, éditeurs. Le dictionnaire des sciences humaines. Paris: Presses Universitaires de France, 2006. p. 893.

${ }^{28}$ AVEN, T. Risk analysis and science. International Journal of Reliability, Quality and Safety Engineering. 2004, 11(1):2.

${ }^{29}$ DUPUY, JP. Complexity and uncertainty: a prudential approach to nanotechnology. In: Allhoff F, Lin P, Moor J, Weckert J, editors. Nanoethics: The ethical and social implications of nanotechnology. New Jersey: John Wiley \& Sons; 2007. p. 119-131.
} 


\section{EMERGÊNCIA DA PLURALIDADE DE FONTES DO DIREITO E DE ATORES NÃO LEGISLATIVOS NA REGULAÇÃO ${ }^{30}$}

O uso de nanotecnologias na produção de medicamentos não encontra resposta do tipo jurídica nas fontes (tradicionais) do direito. Logo, surge a emergência por novas fontes de direito, dentre as quais podemos destacar atores não legislativos, para atuarem na regulação. Um desses atores não legislativos que abordaremos nesse item é a ANVISA. Inicialmente faremos uma contextualização da Agência a fim de situá-la no universo prático e jurídico.

A ANVISA foi criada pela Lei $n^{\circ} 9.782$, de 26 de janeiro de 1999. A referida lei definiu o Sistema Nacional de Vigilância Sanitária e criou a Agência Nacional de Vigilância Sanitária; sua competência abrange a regulação sanitária e a regulação econômica do mercado. A Agência encontra-se vinculada ao Ministério da Saúde e integra o Sistema Único de Saúde (SUS), absorvendo seus princípios e diretrizes. Além da atribuição regulatória, também é responsável pela coordenação do Sistema Nacional de Vigilância Sanitária (SNVS), de forma integrada com outros órgãos públicos relacionados, direta ou indiretamente, ao setor da saúde.

A ANVISA é uma autarquia sob regime especial, ou seja, para fins de direito administrativo, isso significa dizer que é uma agência reguladora caracterizada pela independência administrativa, estabilidade de seus dirigentes durante o período de mandato e autonomia financeira. Na estrutura da Administração Pública Federal, a Agência está vinculada ao Ministério da Saúde, sendo que esse relacionamento é regulado por Contrato de Gestão. 0 referido contrato estabelece que a Agência é responsável pelo controle sanitário da produção e da comercialização de produtos e serviços submetidos à vigilância sanitária, dos ambientes, dos processos, dos insumos e das tecnologias, dentre outras funções.

A(s) resposta(s) para a regulação do tema demanda um (re)processar que considere a emergência da pluralidade de fontes do direito e de atores não legislativos, visto que essas respostas podem vir, inclusive, do sistema da saúde ou da política. E é nesse sentido que se deve observar a relevância do papel regulatório exercido pela ANVISA. As fontes tradicionais do

\footnotetext{
${ }^{30}$ Para esse subitem não serão utilizadas as categorias sistêmicas relacionadas à expectativa jurídica e diferenciação funcional. A escolha teórica para esse ponto foi a pluralidade de fontes e a regulação na ótica portuguesa.
} 
direito (a lei) já não dão conta de regular, por exemplo, itens atrelados à qualidade do medicamento e ao uso da tecnologia nano. As resoluções da agência podem ser vistas como fonte de regulação, tendo em vista que exercem papel vinculante quando o assunto é regulação de medicamentos.

Considerando o abismo epistemológico entre o direito vigente ${ }^{31}$ e as nanotecnologias, vislumbrar a agência como uma fonte oriunda de um ator não legislativo e emergente, para subsidiar a regulação numa perspectiva técnica, é uma possibilidade que deve ser considerada.

Para uma discussão mais clara sobre a questão das fontes oriundas de atores não legislativos, é preciso que apresentemos algumas observações teóricas no que tange à compreensão dessas fontes.

Dentro dessa discussão, é oportuno observarmos as contribuições do direito constitucional português, especialmente no que tange à lei em sentido material e à lei em sentido formal. Assinala Miranda que "a lei em sentido material corresponde à lei como acto da função legislativa [...] e é sempre, necessariamente, também lei em sentido formal. Já não a lei em sentido formal, que pode ou não ser revestida de conteúdo legislativo 32 ". Conforme o autor, a distinção entre ambas tem histórico na doutrina alemã do século XIX. Destaca, ainda, que a abstração, enquanto característica da lei está sendo deixada de lado ou abandonada, devido à emergência das leis-medidas. Pode-se considerar que esse é um fenômeno de administrativização do legislativo, assunção de poderes normativos pelo executivo ${ }^{33}$.

0 executivo, com atribuições de natureza de competência legiferante e diante da complexidade incalculável, esgota a dicotomia lei-regra e lei-medida, e cada vez mais, são tênues as fronteiras entre legislação e administração. A dilatação desses campos e a pulverização decisionista conduzem à descentralização dos poderes normativos, descodificação, deslegalização e desregulação ${ }^{34}$. Nota-se, pois, que é possível um (re)processar que possibilite a regulação oriunda, inclusive, do executivo, se considerarmos que a ANVISA está vinculada ao Ministério da Saúde, então a regulação foi, em certa medida, descentralizada para o executivo.

${ }^{31}$ CARVALHO, Délton Winter. Dano ambiental futuro: a responsabilização civil pelo risco ambiental. Rio de Janeiro: Forense, 2008.

${ }^{32}$ MIRANDA, Jorge. Teoria do Estado e da Constituição. Coimbra: Coimbra Editora, 2002, p. 358.

${ }^{33}$ Merece relevo destacar que, nessa temática, o autor apresenta outros fundamentos para "justificar" a natureza das leis-medidas que não foram apresentadas na presente reflexão. MIRANDA, Jorge. Teoria do Estado e da Constituição. Coimbra: Coimbra Editora, 2002.

${ }^{34}$ MIRANDA, Jorge. Manual de Direito Constitucional. Tomo V. Coimbra: Coimbra Editora, 2010. 

MEDICAMENTOS: O ESTADO DA ARTE DA REGULAÇÃO NO BRASIL

Há um descompasso entre o avanço da nanotecnologia e a regulação jurídica (quiçá sanitária). O legislativo e o direito ainda não dão conta de enfrentar a criatividade e evolução humana quando o assunto é tecnologia em nano escala.

Em função desse descompasso, é crucial pensarmos no diálogo entre as diversas fontes do direito, isso é imprescindível para as nanotecnologias. É preciso alargar a noção de regra jurídica, ou seja, ir além do texto da lei para enfrentar a realidade das nanotecnologias na produção de medicamentos. Em tempos de globalização, de nanotecnologias e de pluralismo de formas de ingresso no cenário jurídico, é indispensável que haja uma releitura da regra jurídica e das suas fontes.

Engelmann ${ }^{35}$ adverte, ainda, que o foco nos aspectos vinculados à regulação não deve preencher os pressupostos do suporte fático (na perspectiva Pontiana), mas sim observar o presente e o futuro, ou seja, no caso das nanotecnologias, observar as regras da ISO (International Organization for Standardization), considerar e valorar as “boas práticas” que apresentam um caráter normatizador em âmbito nacional/internacional, bem como, as normatizações (sem caráter eminentemente jurídico na acepção clássica das fontes) que fogem à concepção clássica de fontes de direito.

Saraiva $^{36}$, notadamente no que tange à questão do universo ISO e a auto-regulação, observa que a incipiente regulação desse setor é uma possibilidade bastante ajustada, mas é necessário (re)pensarmos, para o futuro, novos mecanismos a serem reequacionados, tais como a mudança de um estilo soft law para o hard law, tendo em vista que a indústria pode acautelar essa autoregulação da ISO. É preciso, também, observar o timing da regulação, seguido do seu conteúdo, extensão, forma e intensidade.

São oportunas as contribuições de Góes e Engelmann ${ }^{37}$ no que se refere à regulação: os autores advertem-nos para a observação da existência do gênero regulação e para as espécies regulação tradicional ou legal e a regulação não tradicional. No Brasil, tendo em vista a ausência de dispositivo legal que contemple as nanotecnologias, estamos diante da não

\footnotetext{
${ }^{35}$ ENGELMANN, Wilson. 0 diálogo entre as fontes do Direito e a gestão do risco empresarial gerado pelas nanotecnologias: construindo as bases à juridicização do risco. In: STRECK, Lenio Luiz; ROCHA, Leonel Severo; ENGELMANN, Wilson (Orgs.). Constituição, Sistemas Sociais e Hermenêutica: Anuário do Programa de Pós-gradução em Direito da UNISINOS: Mestrado e Doutorado. Porto Alegre: Livraria do Advogado, 2012, n.9, p. 319-344.

${ }^{36}$ SARAIVA, Rute. "Memórias de um átomo": nanotecnologia, percepção do risco e regulação. In: GOMES, Carla Amado. (Org.) Direito(s) dos riscos tecnológicos. Lisboa: Associação Académica da Faculdade de Direito de Lisboa, 2014. p. 485-534.

37 ENGELMANN, Wilson; GÓES, Maurício de Carvalho. Direito das Nanotecnlogias e o Meio Ambiente de Trabalho. Porto Alegre: Livraria do Advogado, 2015.
} 
regulação. Nota-se, pois, que a partir dessa classificação de regulação proposta pelos autores, pode-se pensar na regulação não tradicional oriunda de outros instrumentos que não sejam a lei, mas sim da regulação da ANVISA.

A lógica verticalizada kelseniana da pirâmide para as fontes do direito (modelo escalonado) não dará conta de enfrentar essas complexidades e riscos mencionados acima. Engelmann ${ }^{38}$ sugere a substituição do modelo verticalizado por um horizontalizado, no qual as fontes sejam postas lado a lado, cujo filtro maior seria a constituição.

Essa releitura das fontes é adequada ao contexto do risco que traz como consequência a incerteza. Na modernidade, já não é mais possível termos certeza e previsibilidade no sistema do direito. Os modelos de gestão (dentre os quais se destaca a Agência Nacional de Vigilância Sanitária) dão respostas regulatórias que o déficit legislativo ainda não consegue dar. Trata-se, pois, de um modelo construtivista da autorresponsabilidade e autogestão do risco. Juridicizar o risco significa lidar com o futuro e com o improvável. Para incorporar o risco à juridicidade é necessário valorar a “juridicização do risco", realizada pelos mecanismos de gestão e autorresponsabilidade do risco ${ }^{39}$. Juridicizar o risco é o desafio imposto ao direito, à saúde e à política.

Engelmann e Flores ${ }^{40}$ nos advertem que

O caminho a ser percorrido em razão do surgimento das nanotecnologias deverá afastar-se das principais características dogmáticas do positivismo jurídico a fim de viabilizar uma atividade criadora, transdisciplinar, dados os contornos inusitados da matéria trabalhada: criatividade [...]

O direito pautado na "velha" dogmática não consegue dar respostas à criatividade humana. A regulação da "criatividade” deve ser tão criativa quanto ela própria na sua essência. É preciso construir respostas para o problema da falta de regulação.

\footnotetext{
${ }^{38}$ ENGELMANN, Wilson. 0 diálogo entre as fontes do Direito e a gestão do risco empresarial gerado pelas nanotecnologias: construindo as bases à juridicização do risco. In: STRECK, Lenio Luiz; ROCHA, Leonel Severo; ENGELMANN, Wilson (Orgs.). Constituição, Sistemas Sociais e Hermenêutica: Anuário do Programa de Pós-gradução em Direito da UNISINOS: Mestrado e Doutorado. Porto Alegre: Livraria do Advogado, 2012, n.9, p. 319-344.

${ }^{39}$ ENGELMANN, Op Cit., 2012.

40 ENGELMANN, Wilson; FLORES, André Stringhi. As nanotecnologias e os marcos regulatórios. Desafios éticos e possibilidades legais para a construção de um direito que normatize as pesquisas. Revista Jurídica, ed. 61, sem numeração. Disponível em: http://revistavisaojuridica.uol.com.br/advogados-leisjurisprudencia/44/artigo162268-1.asp . Acesso em 24/08/2015.
} 
A partir de uma perspectiva crítica ${ }^{41}$, pode-se questionar se é possível gerenciar o risco oriundo dessa "criatividade", com base no atual ordenamento jurídico, e mais: como inserir no direito os aspectos ou as contribuições das normatizações que ocorrem fora do universo eminentemente jurídico? Quais seriam os acoplamentos estruturais entre direito e saúde que permitiriam a regulação do uso de tecnologia nano na produção de medicamentos? $E$ mais (conforme Engelmann ${ }^{42}$ ): Como regular? 0 que regular? E por que regular?

Nesse contexto, a Constituição pode servir como sustentáculo para a ideia trazida por Von Hohendorff e Engelmann ${ }^{43}$ de diferentes fontes do direito, tanto nacionais quanto internacionais, de origem do Estado ou de outros atores. Somente assim é possível formar uma "árvore da decisão". Desse modo, o direito poderá dar respostas jurídicas adequadas ao contexto da (r)evolução científica, na qual está o uso de tecnologia nano. Essa possibilidade permite um marco regulatório que dê conta das complexidades inerentes às nanotecnologias. Um marco regulatório tradicional, calcado na lei, com conceitos fixos não se mostra adequado à velocidade das transformações das ciências duras.

Cabe observar, no contexto da pluralidade de fontes, que o paradigma regulatório é um instrumento fundamental da política de saúde nos países ocidentais e deve ser também perspectivada como uma nova área científica e académica ${ }^{44}$. A teoria da regulação emerge de vários ramos do conhecimento, dentre os quais o do direito, o da economia, o da saúde e demanda reequacionar os interesses sociais e jurídicos na atual conjuntura. Assim, a lei deve ser a base com conceito alargado que estará no plano da legislação em geral e que servirá de base para a regulação.

Tem-se, no Brasil, um conceito alargado de direito à saúde, insculpido no art. 196, da Constituição Federal de 1988, e as próprias leis que instituíram a Vigilância Sanitária e a ANVISA (leis 6.360/1976 e 9.782/1999, respectivamente), conforme já referido anteriormente. No entanto, é preciso reequacionar as ações para a regulação intersetorial, bem como rever o monopólio da lei como sendo a única fonte reguladora e possibilitar o ingresso de novos atores não legislativos no cenário da regulação, como, por exemplo, a ANVISA.

\footnotetext{
${ }^{41}$ É oportuno destacar que esse questionamento, em especial, toma por base a influência do pensamento sistêmico de Niklas Luhmann.

${ }^{42}$ ENGELMANN, Op Cit., 2012

${ }^{43}$ VON HOHENDORFF; ENGELMANN, Op Cit., 2014.

${ }^{44}$ NUNES, Rui. Regulação da saúde. Porto: Vida Económica, 2014.p.13.
} 


\section{A PRODUÇÃO DE MEDICAMENTOS COM PARTÍCULAS NANO: O ESTADO DA ARTE DA (DES)REGULAÇÃO DA NANOTECNOLOGIA NA PRODUÇÃO DE MEDICAMENTOS NO BRASIL}

Toca ao Sistema Nacional de Vigilância Sanitária ${ }^{45}$ a função de regular a entrada de medicamentos no mercado, assim como fiscalizar e monitorar suas condições de produção, prescrição, comercialização, transporte, armazenamento e a ocorrência de eventos adversos advindos de seu uso ${ }^{46}$.

O uso de tecnologia nano para a produção de medicamentos eficazes e seguros é uma estratégia positiva para a saúde pública, tendo em vista a relação custo/benefício. Todavia, temos dois obstáculos: a falta de informações acerca dos riscos e a ausência de regulação.

No que tange à falta de informação, essa já foi abordada nos apontamentos iniciais relacionados ao risco. Sobre a (des)regulação, é preciso considerar a ideia de reforma dos sistemas, em especial do direito e da saúde, para o enfrentamento da regulação do uso de tecnologia nano na produção de medicamentos. A reforma é uma necessidade vital para a regulação. Vejamos as contribuições de Vial $^{47}$ acerca da discussão da reforma:

[...] a "reforma" se torna de fato uma necessidade [...] A grande questão é que a reforma pressupõe crise; esta, por sua vez, muitas vezes é "reformada" através de uma nova legislação, criando, assim, um círculo vicioso crise-reforma-lei. Porém, o problema permanece, pois não efetivamos uma reforma dos próprios reformadores. Observamos no sistema da política brasileira uma constante irritação quanto à criação de novas legislações.

\footnotetext{
${ }^{45}$ Cumpre destacar que a Agência Nacional de Vigilância Sanitária (ANVISA) criou o Comitê Interno de Nanotecnologia da Agência Nacional de Vigilância Sanitária - ANVISA - para enfrentar o tema da nanotecnologia, por meio da portaria de 1.358, de 20 de agosto de 2014.

${ }^{46}$ ALMEIDA FILHO, Naomar. O conceito de saúde e a Vigilância Sanitária: notas para a compreensão de um conjunto organizado de práticas de saúde. In: Costa $\mathrm{AE}$, organizadora. Vigilância Sanitária desvendando o enigma. Salvador: EDUFBA, 2008, p. 19-43.

${ }^{47}$ VIAL, S. R. M. O Sistema da Saúde e o Sistema da Educação: uma reflexão sobre as expectativas e a constante necessidade de reforma. In: Lenio Luiz Streck; Leonel Severo Rocha; Wilson Engelmann. (Org.). Constituição, sistemas sociais e hermenêutica. Anuário do Programa de Pós-Graduação em Direito da Unisinos. 10 ed. São Leopoldo: Livraria do Advogado, 2013, p. 221-241.p. 231.
} 
A reforma jurídica e sanitária, para enfrentar a produção de medicamentos com nanotecnologia, tem ocorrido de modo muito lento e pouco satisfatório no Brasil, por isso anteriormente mencionamos o déficit legislativo. Na sociedade atual, o sistema do direito tem que dar respostas que ultrapassam as respostas tradicionais, assim, percebemos que a demanda da regulação do uso de tecnologia nano na produção de medicamentos não encontra resposta no direito fundamentado na perspectiva dogmática, a reforma legislativa não tem dado conta de responder à complexidade do tema. Essas "novas legislações” não ultrapassam o campo de discussão, não se tornam “decisões coletivamente vinculantes” (leis). O processo de reforma, no âmbito do tema proposto, é extremamente engessado. É preciso que tomemos a sério a demanda de efetivar o Direito à Saúde e de modo mais amplo, atentando, também, para a necessidade de (re)construir o direito regulatório.

A reforma da regulação brasileira, por meio de lei, iniciou com o Projeto de Lei $n^{\circ} 5.076$, de 2005, que procurou regulamentar a pesquisa e o uso da nanotecnologia no país, criou uma Comissão Técnica Nacional de Nanossegurança (CTNano), e instituiu um Fundo de Desenvolvimento de Nanotecnologia (FDNano) para o enfrentamento do tema ${ }^{48}$, mas ainda assim, na via formal de fonte do direito.

Esse projeto de regulação era amplo, estava focado na regulação do uso de nanotecnologias em diferentes áreas. 0 projeto apresentava medidas regulatórias, tais como o monitoramento dos produtos pelos detentores de seu registro após a comercialização, a necessidade de identificação no rótulo do produto como nanotecnológico e o incentivo às pesquisas de avaliação de risco para mensurar-se e gerir o risco no futuro. ${ }^{49}$

O mais paradoxal é que o projeto foi avaliado por três comissões diferentes da Câmara Federal, (Comissão de Finanças e Tributação, Comissão de Desenvolvimento Econômico, Indústria e Comércio e Comissão de Ciência e Tecnologia, Comunicação e Informática) e todas elas deram pareceres desfavoráveis ${ }^{50}$. Os argumentos foram relacionados à incompatibilidade e inadequação orçamentária e financeira ou falta de consenso entre o Executivo e o setor privado sobre como regulamentar a nanotecnologia no país, ou por receio de que o controle de risco do projeto pudesse inibir os investimentos na área. Nota-se que o legislativo ainda não é capaz de dar uma resposta jurídica adequada à demanda. Regular não é consensuar. Isso só corrobora a

\footnotetext{
${ }^{48}$ BRASIL, Op Cit., 2005.

49 BRASIL. Projeto de Lei no 5.076, de 2005. Deputado Edson Duarte. Brasília, DF. 2005. Disponível em: http://www.camara.gov.br/sileg/integras/297210.pdf.

${ }^{50}$ Brasil, Op Cit., 2005.
} 
ideia e a necessidade da emergência da pluralidade de fontes do direito e de atores não legislativos.

Os próprios relatores citaram a ANVISA como responsável pela regulação na seara da saúde. É mais uma "abertura” para a pluralidade das fontes e dos atores não legislativos.

No que tange aos aspectos da rotulagem, os relatores do Projeto de Lei $n^{\circ} 5.076$, de 2005, basearam-se no Código de Defesa do Consumidor, na Lei $n^{\circ} 8.078 / 91$ e no Decreto $n^{\circ}$ 4.680/03 que determina os critérios para rotulagem de alimentos que possuam OGM (Organismos Geneticamente Modificados) para inferir que, por analogia, os produtos nanotecnológicos sejam corretamente identificados. Todavia, observa-se que essa analogia não está funcionando e que atualmente há, no mercado, produtos nanotecnológicos sem identificação. O PL foi arquivado em 28 de novembro de 2008.

No ano de 2010, foi elaborado o Projeto de Lei do Senado $n^{\circ} 131$, que objetivava abarcar as questões de identificação do uso de tecnologia nano, por meio de símbolos e expressões, representando o uso de tecnologia nano nos alimentos, nos cosméticos, nos saneantes, nos medicamentos e nos correlatos. Pouco tempo depois, em 2013, esse projeto foi rejeitado pela Comissão de Assuntos Sociais (CAS) e pela Comissão de Meio Ambiente, Defesa do Consumidor e Fiscalização e Controle (CMA). Nota-se que ainda há muita resistência do sistema da política quando o assunto é regular (tomar decisões coletivamente vinculantes) na área da nanotecnologia, o lobby em torno do tema ainda é muito forte.

O PL n $n^{\circ} 5133 / 2013$ que aborda as questões de rotulagem de produtos da nanotecnologia e de produtos que fazem uso da nanotecnologia, ainda está em tramitação e sem muita discussão.

Destaca-se, ainda, o PL Nº.741/2013 que está em tramitação e dispõe sobre a Política Nacional de Nanotecnologia, a pesquisa, a produção, o destino de rejeitos e o uso da nanotecnologia no país.

0 registro dos medicamentos nanotecnológicos segue a dinâmica de registro de qualquer outro medicamento novo, ou seja, deve ser feito pela ANVISA. Esse processo de registro tem a finalidade de, dentre outras coisas, avaliar a eficácia, a segurança e também o ganho terapêutico do medicamento. Todavia, a falta de regulamentação é um fator que dificulta a regulação dos medicamentos nanotecnológicos por parte da Agência, que por consequência, não tem como saber quais testes devem ser feitos a fim de garantir a segurança desses medicamentos, dificultando, assim, ainda mais o seu registro. ${ }^{51}$

${ }^{51}$ BATISTA; PEPE, Op Cit., 2014. 
Batista e Pepe ${ }^{52}$, em levantamento realizado junto à ANVISA, identificaram, no mínimo, 07 medicamentos nanotecnológicos, registrados no Brasil, até dezembro de 2012. Dos sete, quatro medicamentos, a despeito de serem nanotecnológicos, foram registrados na ANVISA como similares. Conforme a legislação em vigor (Decreto $\mathrm{n}^{\circ} 3.961$, de 10 de outubro de 2001), considera-se medicamento similar quando for equivalente ao registrado. 0 processo de encapsulação dos fármacos, seja por lipossomas, polímeros ou ciclodextrina, altera a biodisponibilidade desses medicamentos nanotecnológicos, quando comparados com o de referência, e é justamente essa diferença tida como sua vantagem. Logo, deveriam possuir atenção especial para o seu registro ou, pelo menos, serem registrados como medicamentos novos, já que esse conceito, na Resolução RDC MS/ANVISA n 136, de 2008, abrange o registro de produtos que possuam alteração na forma farmacêutica, concentração, via de administração e propriedades farmacocinéticas.

Destaca-se, ainda, outro agravante: a falta de uma identificação clara na bula desses produtos quanto às suas constituições nanotecnológicas, inclusive naqueles que foram registrados como medicamentos novos. Na bula (destinada ao paciente) essa informação não consta em nenhum dos produtos, enquanto que naquela destinada aos profissionais há apenas a referência ao tamanho nanométrico daqueles constituídos por lipossomas. As embalagens também não indicam claramente tratar-se de um medicamento nanotecnológico. ${ }^{53}$

Observa-se, pois, que na atual conjuntura de (des)regulação, ainda há dificuldades do tipo jurídicas e práticas, no que tange a encontrar informações sobre a presença de constituintes nanométricos e a não referência a essa característica quando do registro do medicamento, justamente por falta de regulação.

Sant'Ann ${ }^{54}$ identificou outros medicamentos nanotecnológicos comercializados no Brasil: Doxil $\circledast$ (doxorubicin), Amphocil $\circledast$ e Abelcet $®$ (anfotericina B), Rapamune $®$ (sirolimus), Emend $₫$ (aprepitant,MK869), TriCor ${ }^{\circledR}$ (fenofibrate), Megace ES $\circledast$ (megaestrol, acetato), Abraxane ${ }^{\circ}$ (paclitaxel), Elestrin ${ }^{\circ}$ (estradiol). Destaca-se o quão complexa é a tarefa de localizar e identificar os medicamentos que utilizam nanotecnologia; a própria base de consulta da ANVISA é complexa.

\footnotetext{
52 BATISTA; PEPE, Op Cit., 2014.

53 BATISTA; PEPE, Op Cit., 2014.

54 SANT'ANNA, L. S.; FERREIRA, A.P. Patents, drug delivery and public health protection: health risk management for nanopharmaceuticals. J Technol Manag Innov, v. 2, n. 8, 2013, p.107-118.
} 
Conforme informações do Ministério da Ciência, Tecnologia e Inovação, destaca-se que das 608 empresas que utilizam nanotecnologias, 150 desenvolvem nanotecnologias e as áreas principais são a Indústria Química; Petroquímica e a Saúde. ${ }^{55}$

Anteriormente sinalizou-se a dificuldade de enfrentar o problema do risco, do desconhecido em relação ao uso da tecnologia nano na produção de medicamentos. Considerando que não há uma informação clara e precisa de que esses medicamentos são nanotecnológicos, nenhum monitoramento específico tem sido feito com seus usuários, ou seja, não há tabulação de dados e, tampouco, avaliação contínua dos efeitos desses medicamentos, logo, a segurança dos mesmos fica comprometida. Nenhuma das proposições legislativas, no seu texto ou nas justificativas, fizeram menção à necessidade de implementar ou regular algum instrumento para enfrentar o risco.

Tendo em vista a falta de comprovação científica do risco, um dos caminhos para a regulação adotada é utilizar o paradigma do princípio da precaução e a avaliação de risco pensada na dinâmica da casuística, por isso o acompanhamento de cada medicamento é crucial. É necessário bservarmos os modelos implantados pela Agência Reguladora Norteamericana FDA (FDA - Food and Drug Administration) e pela Agência Reguladora EMA (European Medicines Agency).

A partir desses dois modelos, as principais ações contempladas na regulação dos medicamentos nanotecnológicos são: registro como medicamento novo; acompanhamento minucioso do Programa de Farmacovigilância da indústria produtora; efetivação de programas de investigação dos efeitos toxicológicos dos medicamentos nanotecnológicos; comunicação à população e aos profissionais de saúde sobre a nanotecnologia e (a falta de informação sobre) seus efeitos, tanto pela inserção de informações no sítio da ANVISA, como pela utilização de outras estratégias; obrigatoriedade de informar claramente na embalagem e na bula de que se trata de um produto nanotecnológico; programa de monitoramento específico do desenvolvimento clínico e do uso do medicamento pelo produtor. ${ }^{56}$

O "mapa conceitual"57 abaixo apresenta e sintetiza as ideias oriundas de uma regulação, que deve contemplar a necessária passagem pelo risco e pelo dever de informação, no ato de registro.

\footnotetext{
${ }^{55}$ BRASIL, Op Cit., 2015.

${ }^{56}$ BATISTA; PEPE, Op Cit., 2014.

57 BRASIL, Agência Nacional de Vigilância Sanitária. Comitê Interno de Nanotecnologia. Diagnóstico institucional de nanotecnologia. Brasília, 25 de março de 2014 . Disponível em:
} 


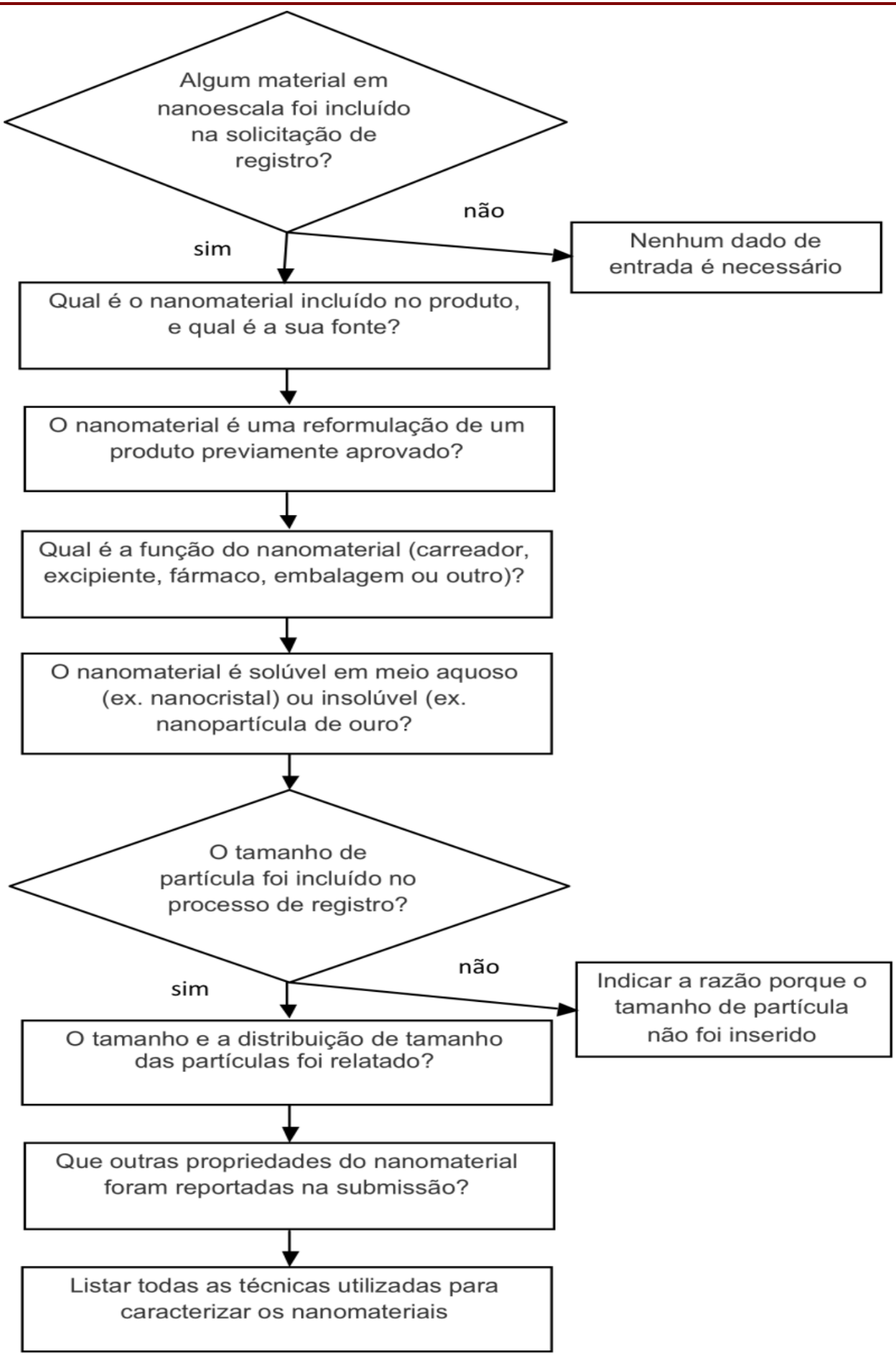

http://portal.anvisa.gov.br/wps/wcm/connect/fb117d80436c3cacb1b5b72a042b41f5/Diagn\%C3\%B3stico+1 nstitucional+de+Nanotecnologia+-+CIN+2014+-+Dicol.pdf?MOD=AJPERES Acesso em 5 jul. 2015. 


\section{CONCLUSÃO}

O sistema do direito está sendo constantemente irritado pelo ambiente para promover as alterações das suas estruturas internas. No entanto, observa-se que as respostas jurídicas que o sistema do direito tem ofertado, mostram-se insuficientes e, portanto, há a necessidade de nova leitura das fontes do direito, talvez com outro olhar teórico para esse aspecto, especialmente no que concerne à pluralidade de atores não legislativos.

A regulação jurídica do uso de nanotecnologias na produção de medicamentos demanda, também, um novo olhar do sistema do direito, da saúde e da política. É preciso (re)construirmos uma cultura de Boas Práticas de Laboratório, destinadas ao manuseio, armazenagem, descarte e segurança pessoal, em ambientes nos quais se trabalha com nanoestruturas. Essa análise de Boas Práticas já existe, mas não no âmbito das nanotecnologias. O direito e a política precisam ser irritados com essas informações a fim de incorporá-las ao seu código binário próprio.

Criar um Banco de Dados, com informações mundiais sobre normas, resoluções, determinações, regulações e dados sobre os efeitos das tecnologias nano, é crucial para a construção dos marcos regulatórios, no âmbito nacional, em função do desconhecimento dos riscos e da própria ausência de coerência, em relação às informações acerca da quantidade de medicamentos produzidos com tecnologia nano.

As dificuldades enfrentadas pela vigilância sanitária, no que concerne à regulação e monitoramento dos eventos adversos (riscos) das novas tecnologias, traz como incremento da complexidade a ausência, a insuficiência ou a incerteza de informações científicas. Nesse contexto, as decisões regulatórias devem levar em conta o princípio da precaução que "propõe o uso controlado dos medicamentos até que novos resultados sobre a avaliação do risco sejam obtidos, de forma a proteger o ambiente e o consumidor". ${ }^{58}$

Existem iniciativas parlamentares visando o estabelecimento de legislações específicas sobre o uso das nanotecnologias, mas ainda é muito incipiente a discussão no âmbito do Congresso Nacional. No que tange à questão da regulação específica de tecnologia nano para

${ }^{58}$ SCHULTE P.A., SALAMANCA-BUENTELLO F. Ethical and scientific issues of nanotechnology in the workplace. Cien Saude Colet, 2007, n. 12, v.5, p.1319-1332 e VON HOHENDORFF R, ENGELMANN W, OSHIROV M. As nanotecnologias no meio ambiente do trabalho: a precaução para equacionar os riscos do trabalhador. Cadernos Ibero-Americanos de Direito Sanitário, 2013, v. 2, n. 2, p.668-683. 


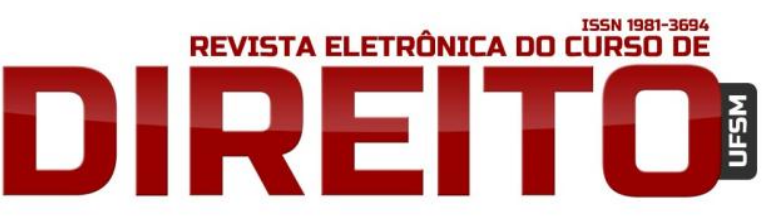

O USO DE NANOTECNOLOGIAS NA PRODUÇÃO DE MEDICAMENTOS: O ESTADO DA ARTE DA REGULAÇÃO NO BRASIL

GABRIELLE JACOBI KÖLLING SANDRA REGINA MARTINI

medicamentos, o estado da arte é ainda pior. Os medicamentos estão no mercado, circulando livremente, mas sem análise técnica específica e, tampouco, com monitoramento dos riscos pósmercado.

Os produtos nanotecnológicos sujeitos à vigilância sanitária que não são identificados ou reconhecidos como tal, pela Agência, em função de não haver norma regulatória específica que exige a informação sobre a natureza nanotecnológica, poderão causar problemas (boa parte deles ainda desconhecidos) de saúde pública. Ainda não temos clareza e certeza quando ao número de medicamentos registrados que utilizam tecnologia nano na sua produção, as bases de dados apresentadas pela Anvisa e pelos pesquisadores da área não idênticas.

É relevante que a VISA acompanhe a evolução regulatória internacional, visto que as agências de regulação dos Estados Unidos e da Europa estão avançadas na seara das boas práticas.

A regulação precisa diferenciar-se ainda mais, pois existe a necessidade de segregar e agrupar os medicamentos produzidos com tecnologia nano em diferentes categorias ou grupos. crucial que se utilize os parâmetros das outras ciências, que não só a jurídica, pois o direito por si só não abarca a variabilidade estrutural e características físico-químicas, que conferem propriedades que podem representar maior ou menor risco sanitário, tais como as nanopartículas solúveis ou insolúveis, por exemplo.

\section{REFERÊNCIAS}

AITH, Fernando. Curso de Direito Sanitário - a proteção do direito à saúde no Brasil. São Paulo: Quartier Latin, 2007.

ALMEIDA FILHO, Naomar. O conceito de saúde e a Vigilância Sanitária: notas para a compreensão de um conjunto organizado de práticas de saúde. In: Costa AE, organizadora. Vigilância Sanitária desvendando o enigma. Salvador: EDUFBA, 2008, p. 19-43.

AVEN, T. Risk analysis and science. International Journal of Reliability, Quality and Safety Engineering. 2004, 11(1):2.

BARRETO, Vicente de Paulo. O Fetiche dos Direitos Humanos e outros temas. 2 ed. Porto Alegre: Livraria do Advogado, 2013. 
BATISTA, Ariane de Jesus Sousa. PEPE, Vera Lúcia Edais. Os desafios da nanotecnologia para a vigilância sanitária de medicamentos. Ciênc. saúde coletiva, v.19, n.7, Rio de Janeiro, Julho de 2014. Disponível em: http://dx.doi.org/10.1590/1413-81232014197.02462013 . Acesso em 13 jun. 2015.

BOURG, D. Principe de précaution. In: Mesure S, Savidan $P$, éditeurs. Le dictionnaire des sciences humaines. Paris: Presses Universitaires de France, 2006.

BRASIL, Agência Nacional de Vigilância Sanitária. Comitê Interno de Nanotecnologia. Diagnóstico institucional de nanotecnologia. Brasília, 25 de março de 2014. Disponível em:

http://portal.anvisa.gov.br/wps/wcm/connect/fb117d80436c3cacb1b5b72a042b41f5/Diagn\%C3\% B3stico+Institucional+de+Nanotecnologia+-+CIN+2014+-+Dicol.pdf?MOD=AJPERES Acesso em 5 jul. 2015.

BRASIL, Agência Brasileira de Desenvolvimento Industrial (ABDI). Panorama Nanotecnologia. Série Cadernos da Indústria ABDI. Volume XIX. Brasília; 2010. Disponível em:

http://www.abdi.com.br/Estudo/PanoramadeNanotecnologia.pdf. Acesso em: 12 ago. 2015.

BRASIL, ANVISA. Agência Nacional de Vigilância Sanitária. Gestão 2005-2010: principais realizações / Agência Nacional de Vigilância Sanitária. Brasília: ANVISA, 2010a.

BRASIL, ANVISA. Agência Nacional de Vigilância Sanitária. Política Vigente para a

Regulamentação de Medicamentos no Brasil, 2004. Disponível em:

http://bvsm.saude.gov.br/bsv/publicacoes/anvisa/manual_politica_medicamentos.pdf. Acesso em: 28 jun. 2015.

BRASIL, Ministério da Ciência, Tecnologia e Inovação. Estratégia Nacional de Ciência, Tecnologia e Inovação 2012-2015. Disponível em:

http://www.mct.gov.br/upd_blob/0218/218981.pdf . Acesso em 22 ago. 2015.

BRASIL. Projeto de Lei ${ }^{\circ}$ 5.076, de 2005. Deputado Edson Duarte. Brasília, DF. 2005.

Disponível em: http://www.camara.gov.br/sileg/integras/297210.pdf.

CARSON, Rachel. Primavera silenciosa. Tradução de Raul de Popillo. São Paulo: Edições

Melhoramentos, 1969. Disponível em

https://biowit.files.wordpress.com/2010/11/primavera_silenciosa_-_rachel_carson_-_pt.pdf .

Acesso em 29 ago. 2015.

CAPRA, Fritjof. O ponto de mutação. Tradução de Álvaro Cabral. São Paulo: Editora Cultrix, 2012. 

MEDICAMENTOS: O ESTADO DA ARTE DA REGULAÇÃO NO BRASIL

CARVALHO, Délton Winter. Dano ambiental futuro: a responsabilização civil pelo risco ambiental. Rio de Janeiro: Forense, 2008.

CHENG. MM. Is the drugstore safe? Counterfeit diabetes products on the shelves. J Diabetes Sci Technol, 2009; 3:1516-20.

CORSI, Giancarlo; ESPOSITO, Elena; BARALDI, Claudio. Glosario sobre la teoría social de Niklas Luhmann. Tradução Miguel Pérez y Carlos Villalobos. México: Anthropos Editorial del Hombre, 1996.

DALLARI, Dalmo Abreu et al. O Direito Achado na Rua. Introdução crítica ao Direito à Saúde. Vol. 4. Brasília: UNB, 2009.

DE GIORGI, Raffaele. 0 risco na sociedade contemporânea. Tradução de Cristiano Paixão. Revista CCJ - UFSC, n. 28, ano 15, junho de 1994, p. 45-54.

DEVALAPALLY, $\mathrm{H}$ et al. Role of nanotechnology in pharmaceutical product development. Journal of Pharmaceutical Sciences, n. 10, v. 96, n. 10, p. 2.547-2.565, 2007.

DUPUY , JP. Complexity and uncertainty: a prudential approach to nanotechnology. In: Allhoff $F$, Lin P, Moor J, Weckert J, editors. Nanoethics: The ethical and social implications of nanotechnology. New Jersey: John Wiley \& Sons; 2007.

ENGELMANN, Wilson; FLORES, André Stringhi. As nanotecnologias e os marcos regulatórios. Desafios éticos e possibilidades legais para a construção de um direito que normatize as pesquisas. Revista Jurídica, ed. 61, sem numeração. Disponível em: http://revistavisaojuridica.uol.com.br/advogados-leis-jurisprudencia/44/artigo162268-1.asp . Acesso em 24 ago. 2015.

ENGELMANN, Wilson; GÓES, Maurício de Carvalho. Direito das Nanotecnlogias e o Meio Ambiente de Trabalho. Porto Alegre: Livraria do Advogado, 2015.

ENGELMANN, Wilson. O diálogo entre as fontes do Direito e a gestão do risco empresarial gerado pelas nanotecnologias: construindo as bases à juridicização do risco. In: STRECK, Lenio Luiz; ROCHA, Leonel Severo; ENGELMANN, Wilson (Orgs.). Constituição, Sistemas Sociais e Hermenêutica: Anuário do Programa de Pós-gradução em Direito da UNISINOS: Mestrado e Doutorado. Porto Alegre: Livraria do Advogado, 2012, n.9, p. 319-344.

FERNANDES, M.F.M., FILGUEIRAS CAL. Um panorama da nanotecnologia no Brasil (e seus macrodesafios). Quim. Nova, v. 8, n. 31, 2008, p.2205-2213. 
FRONZA, T.; GUTERRES, S. S.; POHLMANN, A. R.; TEIXEIRA, H.F. Nanocosméticos: em direção ao estabelecimento de marcos regulatórios. Porto Alegre: Gráfica da UFRGS, 2007.

KANT, Immanuel. La metafísica de los costumbres. Madrid: Tecnos, 1999.

LUHMANN, Niklas. El derecho de la sociedad. México: Universidad Iberoamericana, 1998.

LUHMANN, Niklas. La Sociedad de la Sociedad. Tradução Javier Torres Nafarrate. Cidade do México: Herder Editorial, 2006.

LUHMANN, Niklas. Sociedad y sistema: la ambición de la teoria. Introducción de Ignacio Izuzquiza. Barcelona: Ediciones Paidos Ibérica, 1990.

LUHMANN, Niklas. Sociologia do Direito I. Tradução Gustavo Bayer. Rio de Janeiro: Tempo Brasileiro, 1983.

LUHMANN, Niklas; DE GIORGI, Raffaele. Teoria della società. Milano: Franco Angeli, 1996.

MATURANA, H. R.; VARELA, F. J. A árvore do conhecimento: as bases biológicas do comportamento humano. Tradução de Humberto Mariotti. São Paulo: Athenas, 2011.

MEDEIROS, E.S.; PATERNO L.G.; MATTOSO L.H.C. Nanotecnologia. In: Durán N, Mattoso LHC, Morais PC, organizadores. Nanotecnologia Introdução, preparação e caracterização de nanomateriais e exemplos de aplicação. São Paulo: Artliber, 2006.

MIRANDA, Jorge. Manual de Direito Constitucional. Tomo V. Coimbra: Coimbra Editora, 2010.

MIRANDA, Jorge. Teoria do Estado e da Constituição. Coimbra: Coimbra Editora, 2002.

MORIN, Edgar. Cabeça bem-feita. Tradução Eloá Jacobina. Rio de Janeiro: Bertrand Brasil, 2008.

NUNES, Rui. Regulação da saúde. Porto: Vida Económica, 2014.

NUNES, João Pimentel. Pedidos de Patente sobre Nanotecnologia publicados no $1^{\circ}$ semestre de 2010. INPI/DART/CEDIN/DIESPRO. Alerta Tecnológico n² 29; 2010. Disponível em:

www.inpi.gov.br/menuesquerdo/...em.../alerta_nano_jan_jun_2010.pdf. Acesso em: 10 mai. 2015.

NUNES, João Pimentel Rohem-Santos P. Pedidos de Patente sobre Nanotecnologia publicados no $2^{\circ}$ semestre de 2009. INPI/DART/CEDIN/DIESPRO. Alerta Tecnológico n²0; 2010a. Disponível em: http://www.inpi.gov.br/menuesquerdo/informacao/alerta_nano_20.pdf. Acesso em: 10 mai. 2015. 

MEDICAMENTOS: O ESTADO DA ARTE DA REGULAÇÃO NO BRASIL

PESSOA, Fernando. Mensagem. Disponível em: http://www.cfh.ufsc.br/ magno/mensagem.htm Acesso em 28 ago. 2015.

PIOVESAN, Márcia Franke. A construção política da Agência Nacional de Vigilância Sanitária. [Mestrado] Fundação Oswaldo Cruz, Escola Nacional de Saúde Pública; 2002.

ROSSI-BERGMAN, B. A nanotecnologia: da saúde para além do determinismo tecnológico. Cienc Cult, v. 2, n. 60, 2008, p.54-57.

RUIZ, Adriana; OSÓRIO DE CASTRO, Cláudia. Medicamentos: falando de qualidade. In: Gabriela Costa Chaves, Renata Reis, Veriano Terto Jr. e Cristina Pimenta (orgs). Rio de Janeiro: ABIA, 2008.

SANT'ANNA, L. S.; FERREIRA, A.P. Patents, drug delivery and public health protection: health risk management for nanopharmaceuticals. J Technol Manag Innov, v. 2, n. 8, 2013, p.107-118.

SARAIVA, Rute. "Memórias de um átomo": nanotecnologia, percepção do risco e regulação. In: GOMES, Carla Amado. (Org.) Direito(s) dos riscos tecnológicos. Lisboa: Associação Académica da Faculdade de Direito de Lisboa, 2014. p. 485-534.

SCHULTE P.A., SALAMANCA-BUENTELLO F. Ethical and scientific issues of nanotechnology in the workplace. Cien Saude Colet, 2007, n. 12, v.5, p.1319-1332.

SCHWARTZ, Germano. 0 tratamento jurídico do risco no direito à saúde. Porto Alegre: Livraria do Advogado Editora, 2004.

SOUSA BATISTA, Ariane de Jesus; EDAIS PEPE, Vera Lúcia. Os desafios da nanotecnologia para a vigilância sanitária de medicamentos. Ciência \& Saúde Coletiva, v. 19, n. 7, p. 2105-2114, Rio de Janeiro, 2014.

SWARBRICK, J. Encyclopedia of Pharmaceutical Technology. New York: Informa Healthcare, 2007.

UNESCO. Organisation des Nations Unies pour l'éducation, la science et la culture. Éthique et Politique des Nanotechnologies. Paris, 2007. Disponível em:

http://unesdoc.unesco.org/images/0014/001459/145951f.pdf . Acesso em 23 ago. 2015.

VIAL, S. R. M. O Sistema da Saúde e o Sistema da Educação: uma reflexão sobre as expectativas e a constante necessidade de reforma. In: Lenio Luiz Streck; Leonel Severo Rocha; Wilson Engelmann. (Org.). Constituição, sistemas sociais e hermenêutica. Anuário do Programa de 
ISSN 1981-3694

(DOI): $10.5902 / 1981369419689$ REVISTA ELETRÔNICA DO CURSD DE

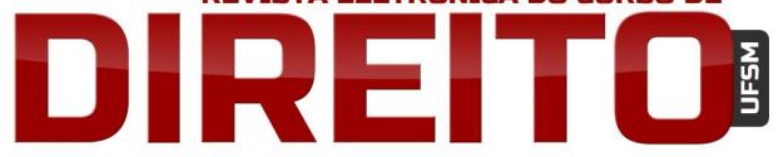

O USO DE NANOTECNOLOGIAS NA PRODUÇÃO DE MEDICAMENTOS: O ESTADO DA ARTE DA REGULAÇÃO NO BRASIL

GABRIELLE JACOBI KÖLLING SANDRA REGINA MARTINI

Pós-Graduação em Direito da Unisinos. 10 ed. São Leopoldo: Livraria do Advogado, 2013, p. 221 241.

VON HOHENDORFF R, ENGELMANN W, OSHIROV M. As nanotecnologias no meio ambiente do trabalho: a precaução para equacionar os riscos do trabalhador. Cadernos Ibero-Americanos de Direito Sanitário, 2013, v. 2, n. 2, p.668-683.

VON HOHENDORFF, Raquel; ENGELMANN, Wilson. Nanotecnologias aplicadas aos agroquímicos no Brasil. A gestão dos riscos a partir do diálogo entre as fontes do direito. Curitiba: Juruá Editora, 2014.

Recebido em: 29/09/2015 / Revisões requeridas em: 18/11/2015 / Aprovado em: 30/11/2015 